\title{
Salt Induced Change of Gene Expression in Salt Sensitive and Tolerant Rice Species
}

\author{
Letícia. C. Benitez ${ }^{1}$, Luciano C. da Maia ${ }^{2}$, Márcia V. Ribeiro ${ }^{1}$, Camila Pegoraro ${ }^{2}$, José A. Peters ${ }^{1}$, Antonio C. de \\ Oliveira $^{2}$, Ariano M. de M. J. ${ }^{3}$ \& Eugenia J. B. Braga ${ }^{1}$ \\ ${ }^{1}$ Laboratório de Cultura de Tecidos de Plantas, Instituto de Biologia, Departamento de Botânica, Universidade \\ Federal de Pelotas, Campus Universitário S/N, CEP 96010-900, Capão do Leão, RS, Brazil \\ ${ }^{2}$ Centro de Genômica e Fitomelhoramento, Departamento de Fitotecnia, Universidade Federal de Pelotas, Campus \\ Universitário S/N, CEP 96010-900, Capão do Leão, RS, Brazil \\ ${ }^{3}$ Empresa Brasileira de Pesquisa Agropecuária Clima Temperado, Rodovia BR 392, Km 78, Caixa Postal 403, \\ 96001-970, Pelotas, RS, Brazil \\ Correspondence: Eugenia Jacira Bolacel Braga, Laboratório de Cultura de Tecidos de Plantas, Instituto de \\ Biologia, Departamento de Botânica, Universidade Federal de Pelotas, Campus Universitário S/N, CEP \\ 96010-900, Capão do Leão, RS, Brazil. Tel: 55-53-3275-734. E-mail: jacirabraga@hotmail.com
}

Received: June 4, 2013 Accepted: August 5, 2013 Online Published: September 15, 2013

doi:10.5539/jas.v5n10p251

URL: http://dx.doi.org/10.5539/jas.v5n10p251

\begin{abstract}
The aim of this study was to quantify the expression of the SalT, LTP-Plant, CDPK and PP1 genes, described as responsive to salinity in rice genotypes, describe the promoter region of these genes and identify possible cis-elements that may be involved in the induction of gene expression under salinity. The cultivars BRS Bojuru (tolerant) and BRS Agrisul (sensitive) were subjected to 0,12, 24, 36 and $48 \mathrm{~h}$ of exposure to salt. For the analysis of qRT-PCR, two detoxification/defense genes and two cell signal transduction genes were selected. In the identification of cis-elements, a region spanning $1.000 \mathrm{bp}$ in the promoter region of genes was analyzed. The Os01g0348900-SalT gene presented an increase in quantification of the relative expression (QR) of 2.081 times in the sensitive and 63.7 times in the tolerant cultivar. The Os03g0251000-LTP gene showed a contrasting increase of the expression between the cultivars BRS Agrisul $(\mathrm{QR}=11.0)$ and BRS Bojuru $(\mathrm{QR}=1.54)$. The genes Os03g0688300-CDPK and Os02g0820000-PP1 apparently do not show response to salinity, since in most treatments, the $\mathrm{QR}$ values were lower than the control. Thirty-eight cis-elements distributed in the four analyzed genes were identified. Of these, six were found only in the Os01g0348900-SalT gene promoter, suggesting a possible involvement of these cis-elements in the induction of expression of this gene under salinity. Based on these results, it can be concluded that these genes do not maintain a direct relation with salinity tolerance, but with mechanisms that allow acclimation to this condition.
\end{abstract}

Keywords: Oryza sativa L., cis-elements, genomics, transcription, salinity

\section{Introduction}

Rice, Oryza sativa L. is a glycophytic plant, and its exposure to high concentrations of salt is extremely detrimental to its growth and development. Regarding biochemical and physiological levels, salinity changes, among other factors, the functioning of the electron transport chain in mitochondria and chloroplasts, causes increasing in the formation of reactive oxygen species (ROS), which, in excess, cause damage to the membranes (lipid peroxidation), protein oxidation and DNA fragmentation (Mishra, Bhoomika, \& Dubey, 2011).

Genetic control of tolerance to salinity is quantitative, involving a gene network of multiple loci distributed in different regions of the genome, which, once activated, can mitigate the effect of stress. Many research groups have used strategies, such as subtractive hybridization, microarray and SAGE, aiming at identifying a large number of genes expressed in response to salinity, which are deposited in public databases (Cotsaftis et al., 2011; Fan et al., 2013). Kumari et al. (2009) when evaluating the transcriptome of rice genotypes with contrasting tolerance to salinity identified 953 genes responsive to salt. However, the results obtained by microarray technique are quite broad, and some genes are selected as "candidates" to be validated in more specific tests for quantification of expression in response to stress. In order to validate some genes responsive to salinity, the 
genes SalT, LTP-Plant, CDPK and PP1 were selected, which are involved in processes of detoxification/cellular defense and signal transduction. In the present work, these genes had their relative expression under salt stress quantified by Real Time PCR.

The SalT gene encodes for a group of proteins that bind to carbohydrate (lectins). Some authors indicate that the binding of lectins to sugars has an important role in signaling response to abiotic stress, modulating signals in cell membranes and cytoplasm (Van Damme, Lannoo, Fouquaert, \& Peumans, 2004). In rice, this gene was isolated and characterized in plants grown under salt stress, confirming its participation in plant adaptation to conditions of osmotic adversity (Zhang et al., 2000).

Lipid Transfer Proteins (LTPs) have been associated with the movement of lipids though membranes, responses to the attack of various pathogens (Chae et al., 2010), response to cold, abscisic acid, drought and salinity. In tomato, LTP-like gene is expressed specifically in stems, when plants are treated with $\mathrm{ABA}$ and $\mathrm{NaCl}$.

Calcium is a secondary messenger involved in the response to many stimuli and participates as an effector in a large group of proteins described as calcium sensors". Among these proteins, calmodulin, calcium-dependent protein kinases (CDPK) are included, which act interconnecting networks of signal transduction. Within the family of genes that encode CDPK proteins, each isoform often have different role in the biology of the organism. In rice, 29 CDPK genes with different physiological functions were identified (Holder, Ridzuan, \& Green, 2012). Thus, studies are necessary to identify CDPKs responsive to salt stress and/or other types of stress. Furthermore, the phospho protein phosphatase (PPP) have an important role in the regulation of metabolism, cell growth and division, and participate in signaling and gene activation in response to light, hormones and stress (Xu, Jing, Mao, Jia, \& Chang, 2007).

Characterization of the promoter region of plant genes usually begins by the identification and characterization of genes expressed in a particular tissue or under conditions of physiological stress, through studies of gene expression. From this functional characterization, structural studies can be started from the upstream region of the gene (Potenza, Aleman, \& Sengupta-Copalan, 2004). Each gene has a unique combination of cis-element associated to transcription factors (TFs), making the temporal and spatial control of transcription specific. Bureau et al. (1996), when studied the promoter region of SalT gene in rice, identified sequences similar to transposable elements like Castaway and Stowaway, which could have influenced the response of expression of this gene. However, further studies are needed to advance in the identification of cis-elements and TFs involved in the response to salinity.

Therefore, the aim of this study was to quantify the relative mRNA expression level of Os01g0348900 SalT, Os03g0251000-LTP-Plant, Os03g0688300-CDPK- and Os02g0820000-PP1 genes in rice genotypes with contrasting tolerance to salinity, as well as describe the promoter region of these genes, identify potential cis-elements that may be involved in the induction of gene expression under salt stress and indicate molecular markers for assisted selection for salt tolerance.

\section{Material and Methods}

\subsection{Candidate Genes: Selection}

The selection of candidate genes responsive to salinity was made from published data of comparative transcriptome of Pokkali (tolerant) and IR64 (sensitive to salt stress) rice cultivars (Kumari et al., 2009). For a representation of different points of stress metabolism, transcripts EF575947 and EF576356 annotated with ontologies related to detoxification/cellular defense, referring to genes Os01g0348900-SalT induced protein and Os03g0251000-LTP- Plant lipid transfer protein were selected, respectively. For the function of signals transduction, transcripts EF576188 and EF576186, referring to genes, Os03g0688300-CDPK-Putative-Calcium-dependent protein kinase and Os02g0820000-PP1-Putative Serine/threonine protein phosphatase were selected, respectively.

\subsection{Primers}

Sequences from transcribed regions for each gene were obtained from the updated annotation of the rice genome (RAP-DB v.5) and in the Primer Express 3.0 software (Applied Biosystems ${ }^{\circledR}$ ) was used top design primers (Table 1). Prior to the design of primers, alignments were made among the possible isoforms of gene families under study to ensure the specificity of each primer set. As the reaction normalizing gene OsActina (Os03g0718100/AK100267) was chosen which is suitable for rice plant under abiotic stress (Zhang et al., 2009). Subsequently, the stability of the expression of this gene under salt stress was verified and confirmed by the database Genevestigator (https://www.genevestigator.com/gv/). In this database it was not found alterations in 
expression level of the selected genes in conditions of stress and no stress as well as genotypes tolerant and sensitive to salinity.

Table 1. List of primers used in the reactions of qRT-PCR and amplicon size (bp)

\begin{tabular}{lllc}
\hline Gene/Transcript & \multicolumn{1}{c}{ Sequence } & Amplicon \\
\hline SalT & F: 5'TCTGGAACGCTTATCGACGC & $3 '$ & \\
Os01g0348900/AK062520 & R: 5'TGGGAATCAAGGGTGGACG & $3 '$ & 50 \\
\hline LTP & F: 5'TATATATCCGCCAGCAGCATGC & $3 '$ & \\
Os03g0251000/AK104253 & R: 5'CCTCCATGAAGCACGGCAC & 3 & 50 \\
\hline CDPK & F: 5'AGGATGGGCGGATCAGCTAC 3' & \\
Os03g0688300/AK105102 & R: 5'TCCCGGATTTCATCATCGC 3' & 50 \\
\hline Ser/thr PP1 & F: 5'ATCTGCTGGCGCATGTCTTC 3' & \\
Os02g0820000/AK120439 & R: 5'GCGAAAATGCCGGTTAGTCG & 3' & 50 \\
\hline Actina 1 & F: 5'CAGCCACACTGTCCCCATCTA & 3' & \\
Os03g0718100/AK100267 & R: 5'AGCAAGGTCGAGACGAAGGA & 3' & 66 \\
\hline
\end{tabular}

*Tm: $60^{\circ} \mathrm{C}$.

\subsection{Sample Preparation and Statistical Design}

Seeds of BRS Bojuru (subspecies japonica) and BRS Agrisul (subspecies indica) rice genotypes were used as plant material, characterized as tolerant and sensitive to salinity, respectively (Benitez et al., 2010).

The experiment was conducted in a greenhouse with $70 \%$ relative humidity and temperature of $25 \pm 2{ }^{\circ} \mathrm{C}$. The seeds were germinated in $2 \mathrm{~L}$ plastic containers with washed sand as substrate and irrigated with Hoagland nutritive solution (Hoagland \& Arnon, 1938). Fourteen days after the date of sowing (vegetative stage), the plants started to be irrigated daily, alternating nutrient solution and $100 \mathrm{~mL}$ of nutrient solution containing 300 $\mathrm{mM} \mathrm{NaCl}$. After $0,12,24,36$ and $48 \mathrm{~h}$ of exposure to salt stress, the leaves of both genotypes were collected and stored at $-80{ }^{\circ} \mathrm{C}$ in ulltrafreezer for subsequent RNA extraction. The experiment followed a completely randomized experimental design in a $2 \times 5$ factorial arrangement ( 2 genotypes $\mathrm{x} 5$ salinity exposure times) with three biological replicates consisting of five plants per pot, totaling 15 plants per treatment.

\section{4 qRT--PCR Analyzis}

Total RNA was extracted from $0.1 \mathrm{~g}$ of leaf tissue obtained from plants exposed to different treatments with $\mathrm{NaCl}$, using Trizol Reagent (Invitrogen $\mathrm{TM}$ ). The quantity of total RNA was measured by spectrophotometer at 260 and $280 \mathrm{~nm}$, which provides an estimate of the relative purity of the RNA. The quality and integrity of the nucleic acid were evaluated by electrophoresis on $1.5 \%$ agarose gel.

The single strand cDNA synthesis for each sample was produced from the mRNA by using oligo (dT) primer and other reagents from SuperScript FirstStrand System for RT-PCR (Invitrogen ${ }^{\mathrm{TM}}$ ). Finally, single-stranded cDNA quality was confirmed by amplification of transcripts of OsActina using PCR and electrophoresis on 1.5\% agarose gel.

The qRT-PCR analysis were performed on 7500 Real-Time PCR System (Applied Biosystems ${ }^{\circledR}$ ) using SYBR ${ }^{\circledR}$ Green (Invitrogen ${ }^{\mathrm{TM}}$ ). Initially, each set of primer was evaluated by dissociation curve, and only those primers with specific amplicons (with a single peak of dissociation from the strands of the PCR products) and with efficiencies: $100 \%, 95 \%, 102 \%$, and $96 \%$ for SalT, LTP, CDPK and PP1, respectively, were kept, according to the formula:

$$
E=\left[10^{-1 / \text { slope }}\right]
$$

PCR reactions were performed in triplicate, each with a total volume of $20 \mu \mathrm{L}$. The cycling conditions were: renaturation of the samples for $2 \mathrm{~min}$ at $50{ }^{\circ} \mathrm{C}$ and activating Taq DNA polymerase for $10 \mathrm{~min}$ at $95{ }^{\circ} \mathrm{C}$. Subsequently, 40 cycles were performed with three steps $\left(95^{\circ} \mathrm{C}\right.$ for $30 \mathrm{~s}, 60^{\circ} \mathrm{C}$ for $1 \mathrm{~min}$, and $72{ }^{\circ} \mathrm{C}$ for $\left.1 \mathrm{~min}\right)$ and finally a last step at $72{ }^{\circ} \mathrm{C}$ for five min. 


\subsection{Data Analysis}

Relative quantification of differential expression was performed using the comparative threshold cycle method (Livack \& Schmittgen, 2001) using the equation $\mathrm{QR}=2^{-\Delta \Delta \mathrm{CT}}$.

In this paper, instead of using a graph showing only means and errors, we decided to use analysis of variance to verify the existence of differences between the samples analyzed in relation to different QRs. Thus, analysis of variance was performed from the replicates of each treatment. The results were considered statistically significant when $P \leq 0.05$. The mean values were compared by Tukey test at $5 \%$ probability. For the analyzes, the statistical software SAS Learning Edition (2002) was used.

\subsection{Cis-elements Analysis}

The identification of putative cis-elements was made by alignments to the database $O$. sativa deposited in www.microsatellite.org. For this, $1000 \mathrm{bp}$ upstream of the presumed start site of transcription of each gene were analyzed, using as reference the sequences deposited for cultivar Nipponbare at The MSU Rice Genome Annotation Project Database and Resource (http://rice.plantbiology.msu.edu/). Cis-elements with $P \leq 5$ by Z-score test were considered significant.

\section{Results and Discussion}

\subsection{Analysis of variance}

The analysis of variance showed significant statistic difference $(P \leq 0.05)$ in the relative quantification of the expression of all genes analyzed at different exposure times to stress in both genotypes.

\subsection{Relative Quantification ( $R Q$ )}

For SalT gene, the highest increase in relative quantification (RQ) of expression in both genotypes was observed. In BRS Agrisul, the increase in the expression was proportional to the increase of time of exposure to stress. With 48 hours of induction, there was a significant increase of expression estimated at 2,081 times the control treatment $(0 \mathrm{~h})$. In the tolerant cultivar, there was an increase by 63.7 times in SalT gene expression in plants subjected to 24 hours of stress, followed by a decrease in gene expression in other induction times, but the QR values observed were also higher but not statistically significant to those observed in the control treatment (Figure 1).

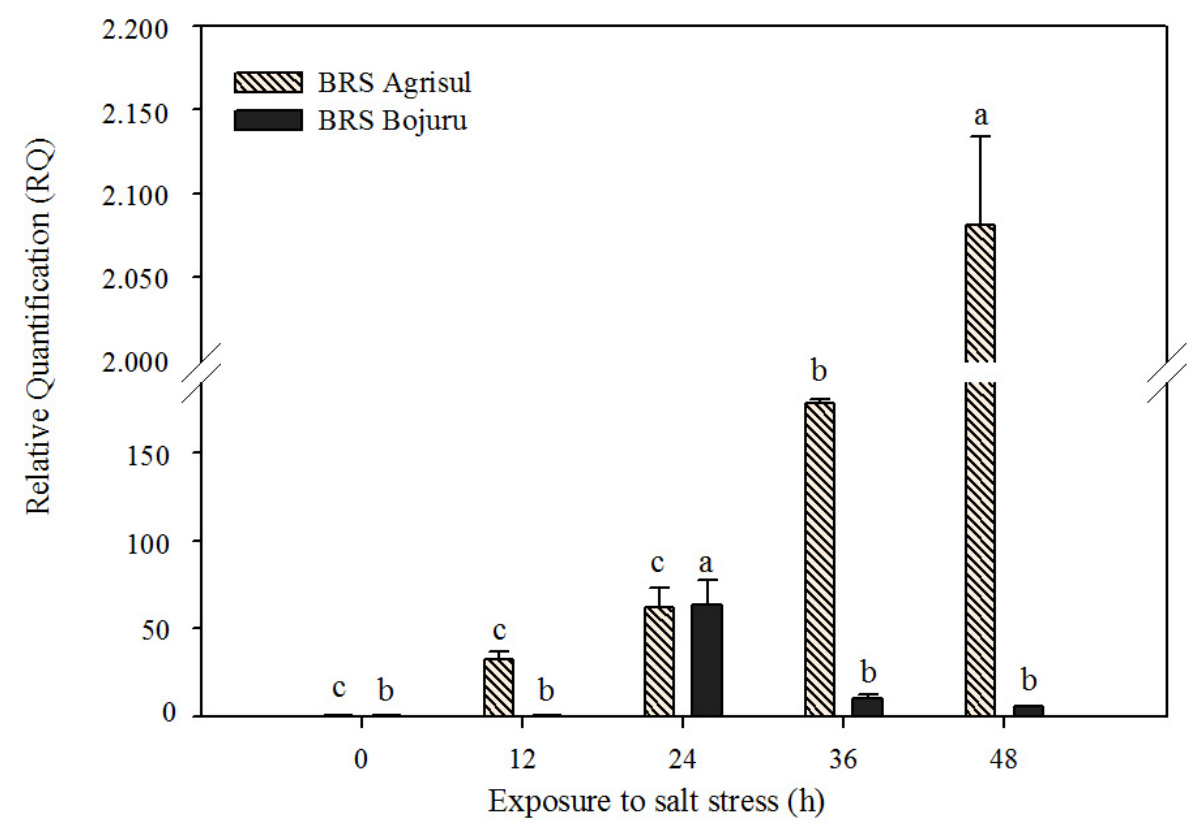

Figure 1. Relative quantification (RQ) of the differential expression of the Os01g0348900 SalT gene, in five periods of exposure to $300 \mathrm{mM} \mathrm{NaCl}$ in BRS Bojuru (tolerant) and BRS Agrisul (sensitive) cultivars. Vertical bars represent the standard deviation of three replicates. Same letter do not differ significantly by Tukey test at $5 \%$ probability 
Although this gene was expressed at a higher level in the sensitive cultivar, it was increased 63 times in the tolerant cultivar, which means that both plants are using this gene in their process of acclimatization. The difference lies in the fact that in the tolerant plant this gene is required only after 24 hours of stress, whereas in the sensitive plant the expression increases proportionally as the stress becomes more severe. Similar results to those found in this work have been reported by Garcia et al. (1998) who observed an increase of SalT gene expression in rice cultivar of indica subspecies, which are typically characterized as sensitive to salinity.

According to Claes et al. (1990), it is not possible to attribute a specific function to the SalT protein, but its function is likely to be of osmoprotection similar to proline, sugars and some small organic acids. Subsequently, Garcia et al. (1998) and Zhang et al. (2000) isolated and characterized the protein SalT in rice plants subjected to salinity, reinforcing the hypothesis that this protein is involved in osmotic adaptation to adverse conditions. Thus, the results of this study demonstrating the increase in SalT gene expression may contribute to the evidence described previously.

The Os03g0251000-LTP gene had an increase in expression after the plants were subjected to 12 hours of stress in both, the sensitive and the tolerant cultivars. Although this increase was significant in both genotypes, it was higher in the sensitive cultivar, similar to the SalT gene, however, to a lesser extent, 11 times compared to control plants. In the tolerant cultivar the increase observed was 1.5 times in the same period of stress exposure (Figure 2). Therefore, these results do not indicate that this gene locus as a major genetic marker of tolerance to saline stress, since it was expressed at a higher level in the sensitive cultivar, however, there are other LTPS loci in the genome of rice, which may have different responses against salt in tolerant genotypes.

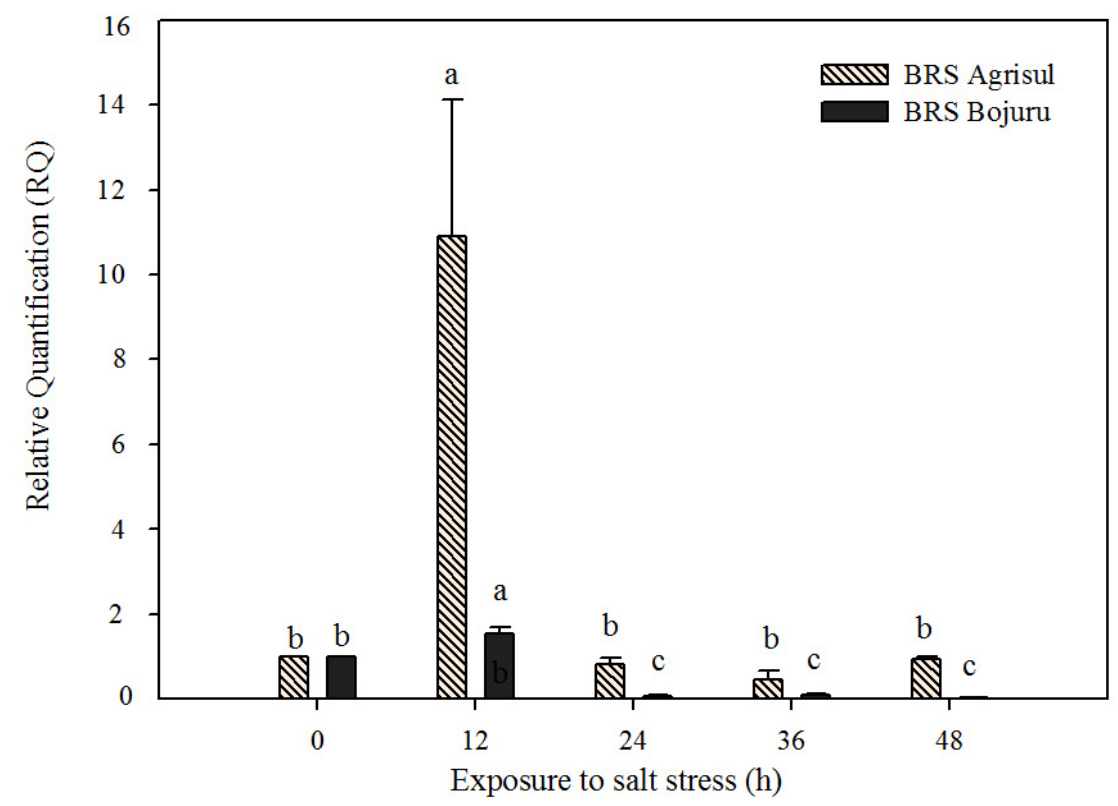

Figure 2. Relative quantification (RQ) of the differential expression of the Os03g0251000-LTP gene in five periods of exposure to $300 \mathrm{mM} \mathrm{NaCl}$ in BRS Bojuru (tolerant) and BRS Agrisul (sensitive) cultivars. Vertical bars represent the standard deviation of three replicates. Same letter do not differ significantly by Tukey test at 5\% probability

The data obtained in this study with the locus Os03g0251000-LTP agree with those found by Wang et al. (2012). When evaluating the expression of different $L T P$ loci of rice genome in plants irrigated with $250 \mathrm{mM} \mathrm{NaCl}$, they observed a significant increase in the expression of Os11g24070, Os04g33920 and Os05g06780 genes within 12 hours of exposure to stress. Moreover, the same authors showed an increase in Os01g60740 gene expression under low temperatures and no changes in transcript levels of this gene under salinity. Thus, this set of information indicates that the LTP gene family plays an important role in rice under adverse environmental conditions, among them salinity. 


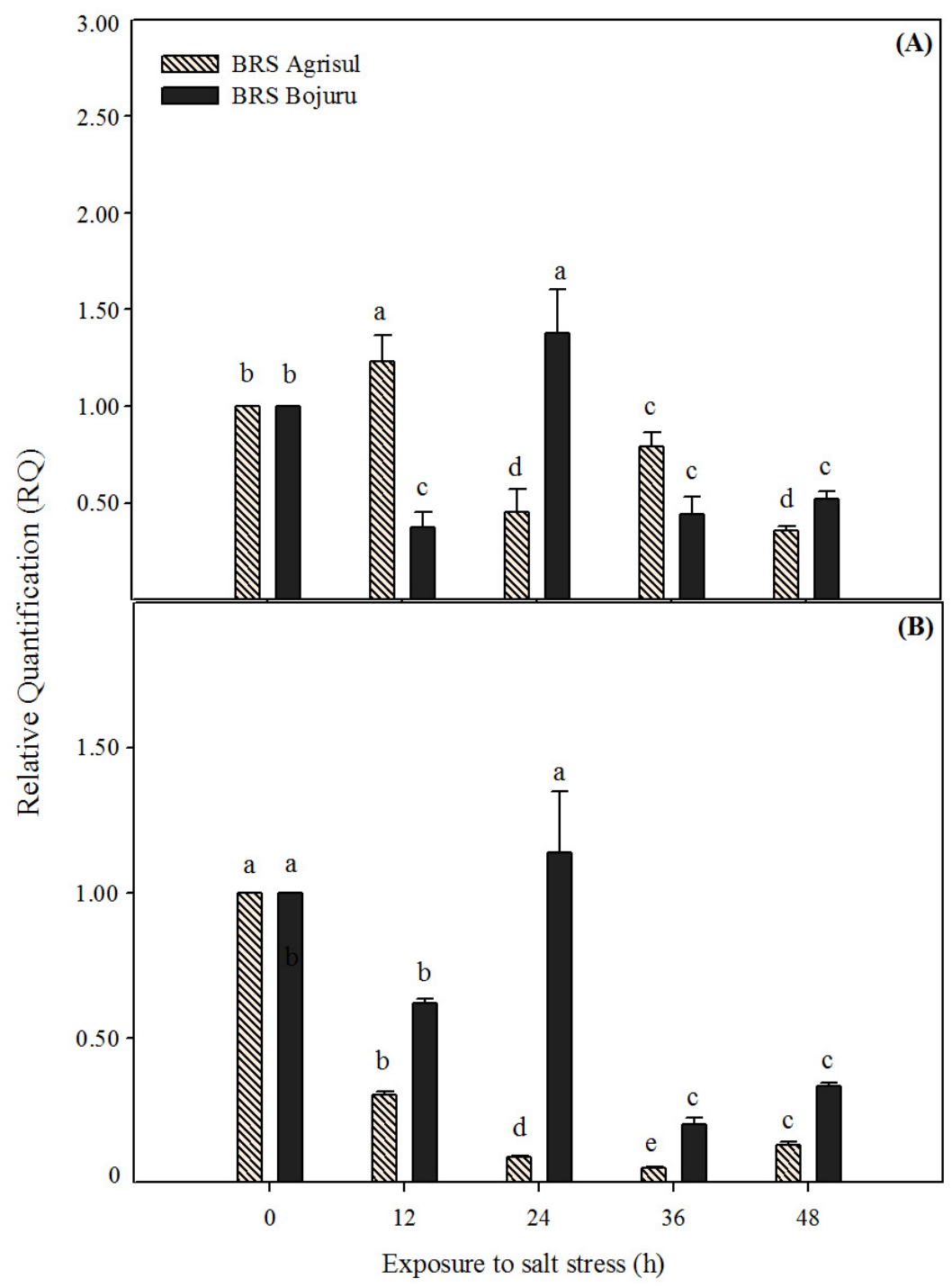

Figure 3. Relative quantification (RQ) of the differential expression of $O s 03 g 0688300-C D P K(\mathrm{~A})$ and Os02g0820000-PPI (B) genes in five periods of exposure to $300 \mathrm{mM} \mathrm{NaCl}$ in BRS Bojuru (tolerant) and BRS Agrisul (sensitive)cultivars. Vertical bars represent the standard deviation of three replicates. Same letter do not differ significantly by Tukey test at $5 \%$ probability

The data obtained from analysis of $0 s 03 g 0688300-C D P K$ gene expression indicate that apparently this locus is not related to salt stress, since in most treatments QR values are lower than those observed in the control treatment in both genotypes evaluated. In tolerant cultivar after 24 hours of stress, there was a slight increase in the expression, $\mathrm{RQ}=1.3$ and the same behavior was observed in the sensitive cultivar after 12 hours of exposure to salt (Figure 3A).

Moreover, Asano et al. (2011) found that in rice Os04t0584600 (OsCDPK12) over expression increased the plants tolerance to salt stress and reduced the accumulation of hydrogen peroxide $\left(\mathrm{H}_{2} \mathrm{O}_{2}\right)$, along with increased expression of OsAPx2 and OsAPx8 enzymes, suggesting that this gene promotes salt tolerance by reducing the accumulation of reactive oxygen species. Also, locus Os03t0128700 (OsCDPK7) over expression showed increased tolerance to cold, salt and dehydration stress (Saijo, Hata, Kyozuka, Shimamoto, \& Izui, 2000). These results associated with those obtained in this experiment indicate that although there is a large number of copies of these genes in the rice genome, possibly not all copies are important to salt stress.

As observed for the Os03g0688300-CDPK gene, no relation with tolerance or adaptive mechanisms to salinity was found for $O s 02 g 0820000-P P 1$ gene. There was a reduction in the expression of this gene for both cultivars, 
except for 24 hours, where the BRS Bojuru cultivar had values close to those found at 0 hours of stress induction (Figure 3B).

In rice, the binding of a protein called RSS1 (Rice Salt Sensitive 1) to the catalytic site of PP1 protein activates/deactivates the cell division and progression of tissue under salt stress, enabling cell growth in contact with the salt. Mutant plants to $r s s 1$ were classified as hypersensitive to salt, possibly due to the non-activation of PP1 proteins and the stop in cell growth in contact with the salt (Ogawa et al., 2011). The results obtained in this study showed a reduction in the expression of Os02g0820000-PP1, indicating that possibly for the cultivars analyzed, this gene is not related to the salt tolerance, however, the possibility that this relations can be observed in other genotypes, organs or periods of exposure to salt cannot be ruled out.

\subsection{Analysis cis-elements}

The analysis of cis-elements allowed the identification of differences in promoter sequences of Os01g0348900-SalT, Os03g0251000-LTP, Os03g0688300-CDPK and Os02g0820000-PP1 genes. A total of 38 cis-elements, distributed along the sequence were identified (Figure 4). From the total cis-elements identified, six (AACACOREOSGLUB1; ACGTABOX; AGCBOXNPGLB; CRTDREHVCBF2; REBETALGLHCB21 and WBOXATNPR1) were found only in the promoter region of the Os01g0348900 SalT gene, which, according to analysis of relative quantification of the expression, showed the highest $\mathrm{QR}$ value after exposure to the salt.

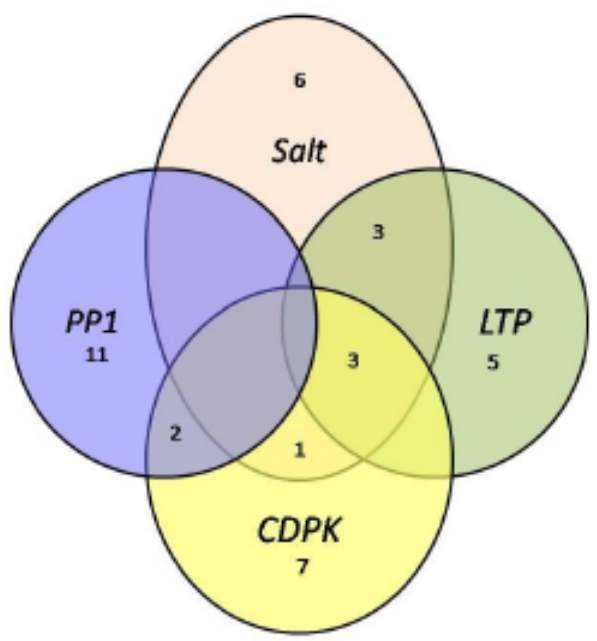

Figure 4. Venn diagram illustrating the distribution of cis-elements in the promoter region (1000 bp) of Osg0348900-SalT, Os03g0251000-LTP, Os03g0688300-CDPK and Os02g0820000-PP1 genes

According to information in the PLACE database, none of these six cis-elements is related to the direct regulation of genes under salt stress. The AGCBOXNPGLB cis-element was identified in Arabidopsis as a sequence that binds to AtERF type proteins, which are responsive to stress conditions. On the other hand, CRTDREHVCBF2 cis-element was identified as a DNA sequence regulated by low temperatures. However, it cannot be ruled out that the presence of these cis-elements modulates in a positive and indirect way the Os01g0348900-SalT gene expression in response to salt stress. However, for this assumption further analyzes are needed, for example, analysis of mutant plants.

According to Lee et al. (2002), the interaction of cis-elements with transcription factors leads to the activation or repression of gene expression in response to environmental or developmental factors. For the Os03g0251000-LTP gene, sequences corresponding to the following cis-elements were found: GCN4OSGLUB1; INRNTPSADB; SEF3MOTIFGM; POLASIG1 and RYREPEATLEGUMINBOX. These cis-elements were not detected in the other genes analyzed in this study.

As possible changes in the sequences of the promoter of this gene are unknown in the BRS Bojuru and BRS Agrisul cultivars, it cannot be ruled out the possibility that the absence of any of these cis-elements in the tolerant cultivar, BRS Bojuru, may be the cause of lower induction of this gene. Sequencing of the promoter 
region of these cultivars to confirm this hypothesis should be carried out. In addition, further analyzes with in silico genes/cis-elements described in salt tolerance can bring more information on this context.

The Os01g0348900-SalT and Os03g0251000-LTP genes share three cis-elements in common in their promoter region, one of them, MYB2AT, is involved in the regulation of genes that are responsive to water deficit. The Os03g0688300-CDPK and Os02g0820000-PP1 genes, which were not induced by salt stress, have two cis-elements (NODCON2GM and OSE2ROOTNODULE) present in their promoter regions. All cis-elements identified in this study are listed in Table 2.

Table 2. List of cis-elements found in the promoter region (1000 bp) of Os01g0348900 SalT, Os03g0251000-LTP, Os03g0688300-CDPK and Os02g0820000-PP1 genes of Oryza sativa

\begin{tabular}{|c|c|c|c|c|}
\hline Cis-element & SalT & $\overline{L T P}$ & $C D P K$ & $P P 1$ \\
\hline AACACOREOSGLUB1 & $1(1.9)^{*}$ & & & \\
\hline ACGTABOX & $1(3.8)$ & & & \\
\hline AGCBOXNPGLB & $1(0.1)$ & & & \\
\hline CRTDREHVCBF2 & $1(1.9)$ & & & \\
\hline REBETALGLHCB21 & $1(1.1)$ & & & \\
\hline WBOXATNPR1 & $3(2.7)$ & & & \\
\hline AMYBOX2 & $1(0.1)$ & $1(0.1)$ & & \\
\hline MYB2AT & $1(2.8)$ & $1(2.8)$ & & \\
\hline TATCCAYMOTIFOSRAMY3D & $1(1.3)$ & $1(1.3)$ & & \\
\hline RYREPEATBNNAPA & $2(2.0)$ & $2(2.0)$ & $2(2.0)$ & \\
\hline RYREPEATGMGY2 & $1(3.8)$ & $2(0.0)$ & $1(3.8)$ & \\
\hline RYREPEATVFLEB4 & $1(0.1)$ & $1(0.1)$ & $1(0.1)$ & \\
\hline NODCON2GM & & & $5(0.1)$ & $4(1.2)$ \\
\hline OSE2ROOTNODULE & & & $5(0.1)$ & $4(1.2)$ \\
\hline TATABOX3 & $1(4.8)$ & & $1(4.8)$ & \\
\hline GCN4OSGLUB1 & & $1(0.0)$ & & \\
\hline INRNTPSADB & & $3(1.0)$ & & \\
\hline POLASIG1 & & $4(0.5)$ & & \\
\hline RYREPEATLEGUMINBOX & & $2(0.1)$ & & \\
\hline SEF3MOTIFGM & & $2(0.1)$ & & \\
\hline ARFAT & & & $1(3.8)$ & \\
\hline CAREOSREP1 & & & $2(0.1)$ & \\
\hline CBFHV & & & $3(0.6)$ & \\
\hline DOFCOREZM & & & $11(4.2)$ & \\
\hline DRE2COREZMRAB17 & & & $1(1.5)$ & \\
\hline LTRECOREATCOR15 & & & $7(2.1)$ & \\
\hline TATAPVTRNALEU & & & $1(0.6)$ & \\
\hline BP5OSWX & & & & $1(0.0)$ \\
\hline CACTFTPРCA1 & & & & $13(3.6)$ \\
\hline CURECORECR & & & & $6(5.2)$ \\
\hline GTGANTG10 & & & & $9(0.4)$ \\
\hline MRNASTA2CRPSBD & & & & $1(0.1)$ \\
\hline P1BS & & & & $1(7.8)$ \\
\hline QARBNEXTA & & & & $1(0.0)$ \\
\hline RAV1AAT & & & & $6(0.0)$ \\
\hline SEF4MOTIFGM7S & & & & $3(2.5)$ \\
\hline T/GBOXATPIN2 & & & & $1(2.0)$ \\
\hline TRANSINITDICOTS & & & & $1(0.9)$ \\
\hline
\end{tabular}

* Values outside the parentheses indicate the number of times that the cis-elements were found in the promoter region and the figures in brackets indicate the value of $P$ according to $Z$-test score. 


\section{Conclusions}

Under the experimental conditions tested, the Os01g0348900-SalT and Os03g0251000-LTP genes apparently are not directly related with salinity tolerance, since they were induced at a higher level in the sensitive cultivar. However, possibly both genotypes require the expression of these genes in different intensities and periods for the process of acclimatization to salinity.

The Os02g0820000-PP1 and Os03g0688300-CDPK genes apparently are not related to tolerance or acclimatization to salinity.

The presence of the cis-elements AACACOREOSGLUB1, ACGTABOX, AGCBOXNPGLB, CRTDREHVCBF2, REBETALGLHCB21 and WBOXATNPR1 may be related to the Os01g0348900-SalT gene expression in response to salt stress, however, further analysis in silico with genes and cis-elements described in tolerance to salt stress, as well as analyzes with mutant plants, can bring more information about this context.

\section{References}

Asano, T., Hayashi, N., Kobayashi, M., Aoki, N., Miyao, A., Mitsuhara, I., ... Ohsugi, R. (2011). A rice calcium-dependent protein kinase OsCPK12 oppositely modulates salt-stress tolerance and blast disease resistance. Plant Journal, 69, 26-36. http://dx.doi.org/10.1111/j.1365-313X.2011.04766.x

Benitez, L. C., Peters, J. A., Bacarin, M. A., Kopp, M. M. Oliveira, A. C. DE., Magalhães Junior, ... Braga, E. J. B. (2010). Salinity tolerance evaluated in genotypes of in vitro cultivated rice. Revista Ceres, 57, 330-337. http://dx.doi.org/10.1590/S0034-737X2010000300007

Bureau, T. E., Ronald, P. C., \& Wessler, S. R. (1996). A computer-based systematic survey reveals the predominance of small inverted-repeat elements in wild-type rice genes. Proceedings of the National Academy of Sciences, 93, 8524-8529

Chae, K., Gonong, B. J., Kim, S. C., Kieslich, C. A., Morikis, D., Balasubramanian, S., \& Lord, E. M. (2010). A multifaceted study of stigma/style cysteine-rich adhesin (SCA)-like Arabidopsis lipid transfer proteins (LTPs) suggests diversified roles for these LTPs in plant growth and reproduction. Journal Experimental Botany, 61, 4277-4290. http://dx.doi.org/10.1093/jxb/erq228

Claes, B., Dekeyser, R., Villarroel, R., Van Den Bulcke, M., Bauw, G., Van Montagu, M., \& Caplan, A. (1990). Characterization of a rice gene showing organ-specific expression in response to salt stress and drought. Plant Cell, 2, 19-27.

Cotsaftis, O., Plett, D., Johnson, A. A. T., Walia, H., Wilson, C., Ismail, A. M., ... Baumann, U. (2011). Root-Specific Transcript Profiling of Contrasting Rice Genotypes in Response to Salinity Stress. Molecular Plant, 4, 25-41. http://dx.doi.org/10.1093/mp/ssq056

Fan, X. D., Jia-Qi Wang, J. Q., Yang, N., Dong, Y. Y., Liu, L., Wang, F. W., ... Li, H. W. (2013). Gene expression profiling of soybean leaves and roots under salt, saline-alkali and drought stress by high-throughput Illumina sequencing. Gene, 512, 392-402. http://dx.doi.org/10.1016/j.gene.2012.09.100. Epub 2012 Oct 12

Garcia, A. B., Engler, J. D. E. A., Claes, B., Villarroel, R., Van Montagu, M., Gerats, T., \& Caplan, A. (1998). The expression of the salt-responsive gene salT from rice is regulated by hormonal and developmental cues. Planta, 207, 172-180.

Hoagland, D. R., \& Arnon, D. I. (1938). The water culture method for growing plants without soil. University of California College of Agriculture, Berkeley.

Holder, A. A., Ridzuan, M. A. M., \& Green, J. L. (2012). Calcium dependent protein kinase 1 and calcium fluxes in the malaria parasite. Microbes and Infection, 14, 825-830. http://dx.doi.org/10.1016/j.micinf.2012.04.006. Epub 2012 Apr 24.

Kumari, S., Sabharwal, V. P., Kushwaha, H. R., Sopory, S. K., Singla-Pareek, S. L., \& Pareek, A. (2009). Transcriptome map for seedling stage specific salinity stress response indicates a specific set of genes as candidate for saline tolerance in Oryza sativa L. Functional and Integrative Genomics, 9, 109-123. http://dx.doi.org/10.1007/s10142-008-0088-5

Lee, T., Rinaldi, N. J., Robert, F., Odom, D. T., Bar-Joseph, Z., Gerber, G. K., ... Simon, I. (2002). Transcriptional regulatory networks in Saccharomyces cerevisiae. Science, 298, 799-804. http://dx.doi.org/10.1126/science.1075090

Livack, K. J., \& Schmittgen, T. D. (2001). Analysis of Relative Gene Expression Data Using Real-Time Quantitative PCR and the 2- ${ }^{\Delta \Delta C T}$ Method. Methods, 25, 402-408. http://dx.doi.org/10.1006/meth.2001.1262 
Mishra, P., Bhoomika, K., \& Dubey, R. S. (2011). Differential responses of antioxidative defense system to prolonged salinity stress in salt-tolerant and salt-sensitive Indica rice (Oryza sativa L.) seedlings. Protoplasma, 250, 3-19. http://dx.doi.org/10.1007/s00709-011-0365-3

Ogawa, D., Abe, K., Miyao, A., Kojima, M., Sakakibara, H., Mizutani, M., ... Takeda, S. (2011). RSS1 regulates the cell cycle and maintains meristematic activity under stress conditions in rice. Nature Communication, 2, 1-11. http://dx.doi.org/10.1038/ncomms1279

Potenza, C., Aleman, L., \& Sengupta-Copalan, C. (2004). Targeting transgene expression in research, agricultural and environmental applications: promoters used in plant transformation. In Vitro Cellular \& Developmental Biology-Plant, 40, 1-22. http://dx.doi.org/10.1079/IVP2003477

Saijo, Y., Hata, S., Kyozuka, J., Shimamoto, K., \& Izui, K. (2000). Over-expression of a single Ca ${ }^{2+}$-dependent protein kinase confers both cold and salt/drought tolerance on rice plants. The Plant Journal, 23, 319-327.

SAS LEARNING EDITION. Programa SAS - Getting started with the SAS Learning Edition. (2002). Cary: SAS Publishing.

Van Damme, E. J., Lannoo, N., Fouquaert, E., \& Peumans, W. J. (2004). The identification of inducible cytoplasmic/nuclear carbohydrate-binding proteins urges to develop novel concepts about the role of plant lectins. Glycoconjugate journal, 20, 449-460.

Wang, H. W., Hwang, S-G., Karuppanapandian, T., Liu, A., Kim, W., \& Jang, C, S. (2012). Insight into the Molecular Evolution of Non-Specific Lipid Transfer Proteins via Comparative Analysis Between Rice and Sorghum. DNA Research, 19, 179-194. http://dx.doi.org/10.1093/dnares/dss003

Xu, C., Jing, R., Mao, X., Jia, X., \& Chang, X. (2007). A wheat (Triticum aestivum) protein phosphatase 2A catalytic subunit gene provides enhanced drought tolerance in tobacco. Annals of Botany, 99, 439-450.

Zhang, W. L., Peumans, W. J., Barre, A., Astoul, C. H., Rovira, P., Rouge, P., ... Van Damme, E. J. M. (2000). Isolation and characterization of a jacalin-related mannose-binding lectin from salt-stressed Rice (Oryza sativa) plants. Planta, 210, 970-978.

Zhang, L. J., Liu, D. H., Wang, Z. H., Yu, C., Cao, J. H., Wang, C. T., \& Jin, D. M. (2009). Expression pattern of GS3 during panicle development in Rice under drought stress: quantification normalized against selected housekeeping genes in real-time PCR. Asian Journal of Plant Sciences, 8, $285-292$. http://scialert.net/abstract/?doi=ajps.2009.285.292

\section{Copyrights}

Copyright for this article is retained by the author(s), with first publication rights granted to the journal.

This is an open-access article distributed under the terms and conditions of the Creative Commons Attribution license (http://creativecommons.org/licenses/by/3.0/). 